\title{
Estimation of SOA formation rate
}

The change in $\mathrm{OA}$ concentration $(\mathrm{COA})$ of the suspended fresh aerosol (prior to photooxidation) is due only to wall-deposition. The wall-loss rate usually exhibits a first-order decay:

$C_{O A}(t)=C_{O A}\left(t_{\text {reference }}\right) \exp \left(\frac{-\Delta t}{\tau}\right)$

Where $\tau$ is the wall-loss time constant, and can be determined by fitting equation (S1) to the decay of the fresh emissions, as shown in Figure S4.

When photo-oxidation is initiated, SOA formation adds mass to the suspended COA, while it continues to decay by wall-deposition. Assuming that $\tau$ does not change much upon SOA formation, SOA concentration in the suspended aerosol can be calculated as the difference between measured $C_{O A}$ and $C_{O A}$ predicted by equation (S1):

$C_{S O A}(t)=C_{O A}(t)-C_{O A}\left(t_{\text {reference }}\right) \exp \left(\frac{-\Delta t}{\tau}\right)$ 
Figures
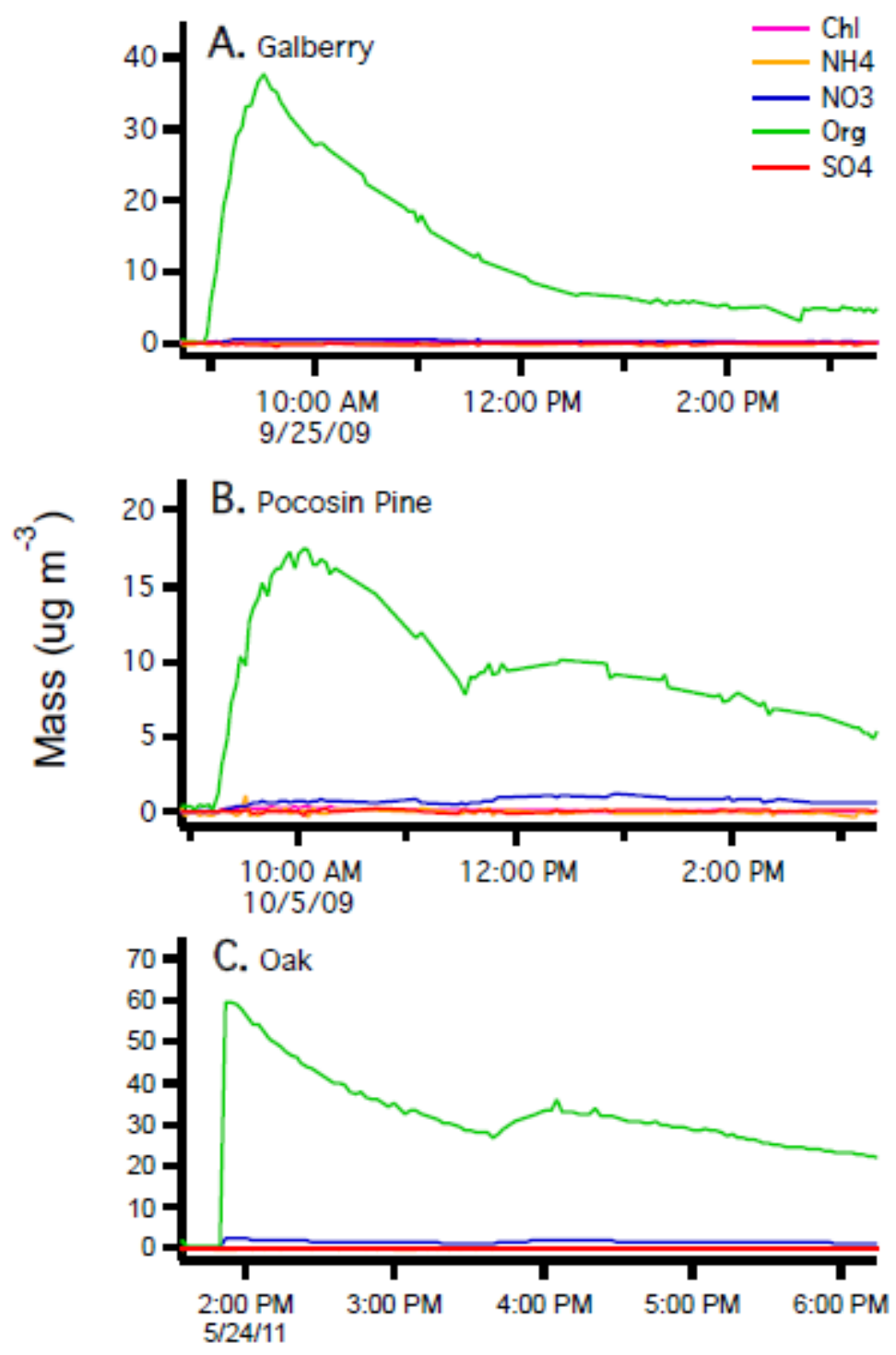

Figure S1 Time series of non-refractory aerosol concentration in biomass-burning emissions measured using an AMS. 


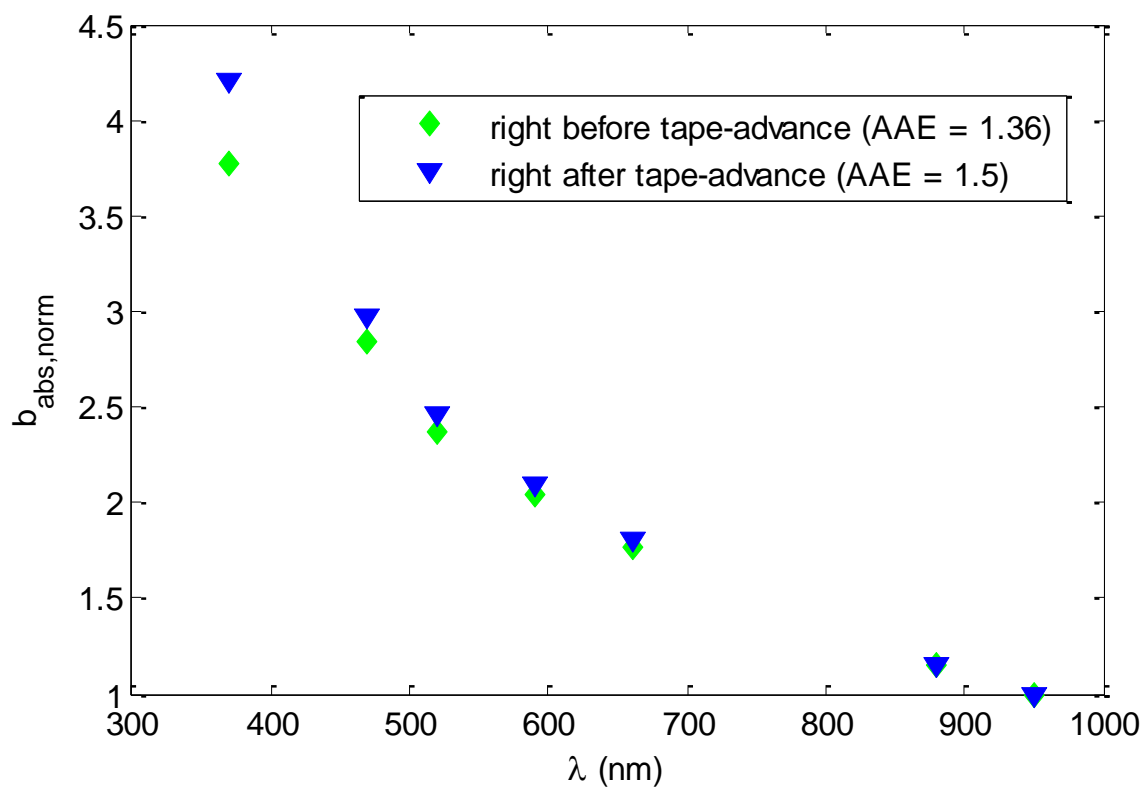

Figure S2 Normalized absorption coefficients obtained from Aethalometer measurements right before and right after tape-advance for oak-burning experiment.

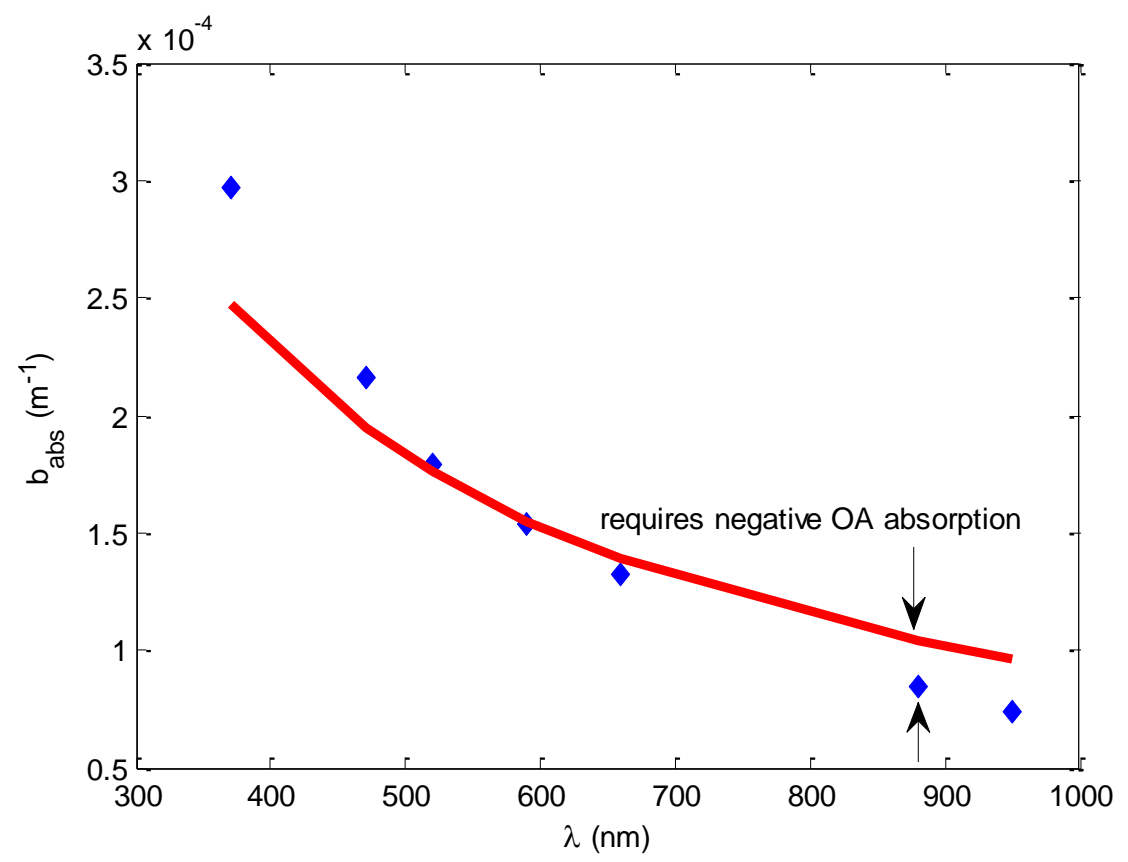

Figure S3 Absorption coefficients obtained from Aethalometer measurements and assuming that $\mathrm{OA}$ absorbs negligibly at $550 \mathrm{~nm}$ for oak POA experiment. The red curve is the absorption 
coefficient of BC. Evidently, the shape of the absorption curve does not allow the assumption of negligible OA absorption at $550 \mathrm{~nm}$, as it would require (unphysical) negative OA absorption at $\lambda<550 \mathrm{~nm}$.

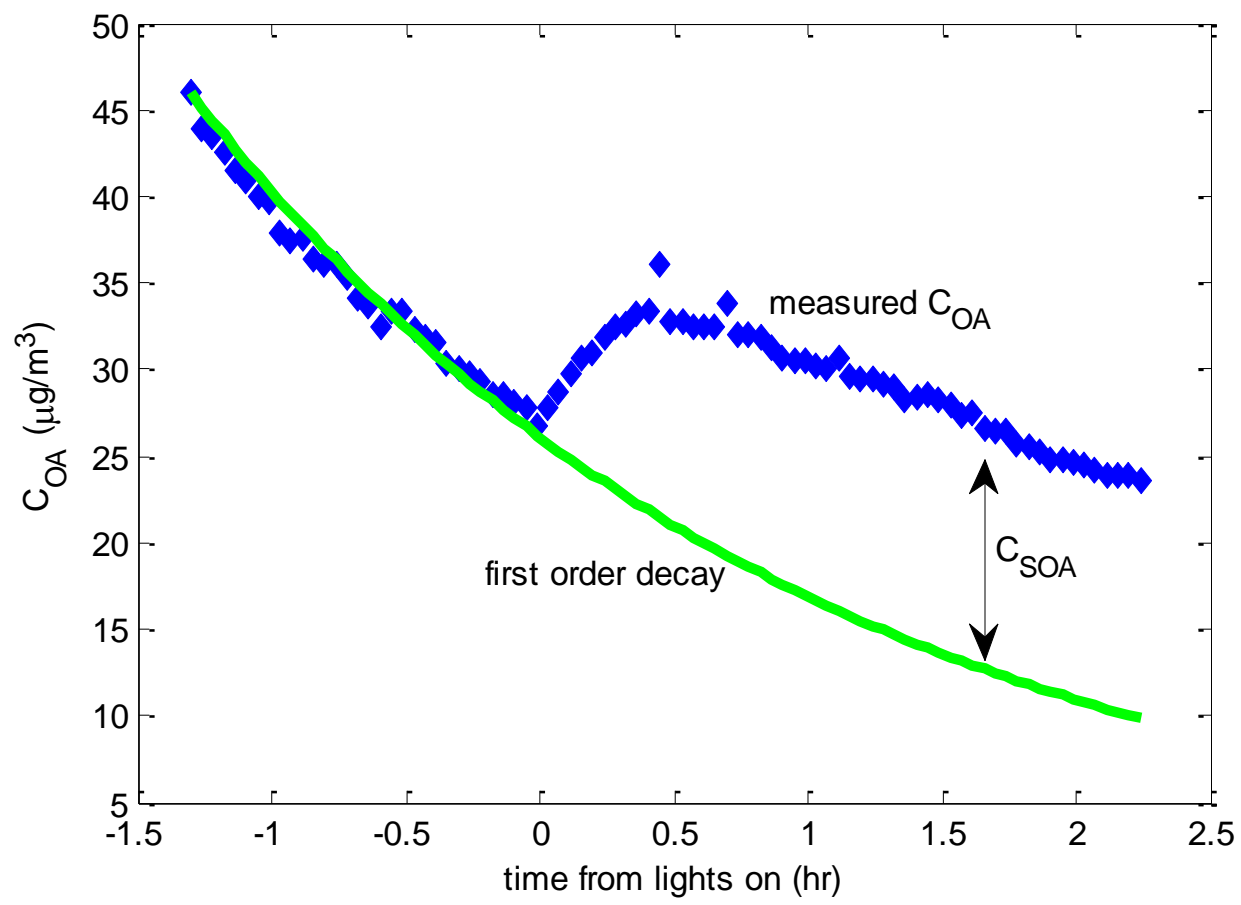

Figure S4 Time series of suspended $C_{O A}$ in oak-aging experiment. The green curve is a fit given by equation (1). The difference between measured $C_{O A}$ and the first order decay fit is the suspended SOA mass concentration (CSOA). 